Research Article

\title{
Approximate Super- and Sub-harmonic Response of a Multi-DOFs System with Local Cubic Nonlinearities under Resonance
}

\author{
Yang CaiJin ${ }^{1,2}$ \\ ${ }^{1}$ Department of Engineering Mechanics, Tsinghua University, Beijing 100084, China \\ ${ }^{2}$ Traction Power State Key Laboratory, Southwest Jiaotong University, Chengdu 610031, China \\ Correspondence should be addressed to Yang CaiJin, ycj78_2012@163.com
}

Received 15 June 2012; Accepted 1 October 2012

Academic Editor: Livija Cveticanin

Copyright (C) 2012 Yang CaiJin. This is an open access article distributed under the Creative Commons Attribution License, which permits unrestricted use, distribution, and reproduction in any medium, provided the original work is properly cited.

\begin{abstract}
A multi-degree-of-freedom dynamical system with local cubic nonlinearities subjected to super/subharmonic excitation is considered in this paper. The purpose of this paper is to approximate the nonlinear response of system at super/sub harmonic resonance. For many situations, single resonance mode is often observed to be leading as system enters into super/sub harmonic resonance. In this case, the single modal natural resonance theory can be applied to reduce the system model and a simplified model with only a single DOF is always obtained. Thus, an approximate solution and the analytical expression of frequency response relation are then derived using classical perturbation analysis. While the system is controlled by multiple modes, modal analysis for linearized system is used to decide dominant modes. The reduced model governed by these relevant modes is found and results in an approximate numerical solutions. An illustrative example of the discrete mass-spring-damper nonlinear vibration system with ten DOFs is examined. The approximation results are validated by comparing them with the calculations from direct numerical integration of the equation of motion of the original nonlinear system. Comparably good agreements are obtained.
\end{abstract}

\section{Introduction}

In engineering, many dynamical systems consisting of large complex components with local physical nonlinearities are found everywhere. For example, in structural dynamics, finite element analysis is often used to obtain accurate discrete models of continuous systems, usually with hundreds of DOFs. If a nonlinear component, such as a joint or a crack, is added to the finite element model, then the system is wholly nonlinear. Other examples of these systems are a pipeline supported by stiffening springs and the exhaust of a road vehicle in which dry friction hinges. A great class of nonlinearities in most of these systems is of cubic 
type. A typical cubic-type nonlinear system is known as Duffing system [1]. Duffing system has many physical applications seen in the literature [2].

The super or subharmonic responses of a dynamical system have been received much attentions. As examples, one can refer to the literature [3]. Other related studies are found in [4-8]. In [4], Ji and Hansen used the averaging method to research a periodically excited nonlinear oscillator with a piecewise nonlinear-linear characteristic and derived an approximate solution for the superharmonic resonance. The validity of the developed analysis was confirmed by comparing the approximate solutions with the results of direct numerical integration of the original equation. Elnaggar and El-Bassyouny [5] studied harmonic, subharmonic, superharmonic, and combination resonances of the additive type of self-excited two coupled-second order systems subjected to multifrequency excitation. The theoretical results were obtained by the multiple-scales method. The same method was used by Eissa and El-Bassyouny [6] to research the nonlinear rolling response of a ship in regular beam seas. The steady-state amplitude and phase of harmonic oscillations for primary and superharmonic resonance were constructed in his work. The harmonic balance method was used to analyze super or sub harmonic response of torsional system with two DOFs in the literatures $[7,8]$.

The objective of this paper is to seek an approximate solution, especially analytic solution, of a MDOFs' system with local nonlinearities under super or sub harmonic resonance conditions. Though the analytic methods mentioned in [3-8], such as the averaging method, the multiple scales method and the harmonic balance method can produce the analytical solutions for the system and give a stability analysis about the solutions; they are often applied to nonlinear systems with low DOFs [4-8]. For large systems, especially large-scale systems, the use of analytic methods results in heavy algebraic manipulation and high-dimensional nonlinear equations. Many challenging problems [9], for example, ill-conditioned iterative matrix and serious stiffness problem, are in solving large nonlinear equations, and special numerical algorithms [10] are required. Consequently, analytic methods often lose their performances for high-dimensional dynamical system. Here, the author attempts to provide with a way to give super/sub harmonic solutions of large nonlinear dynamical systems in this paper. For this purpose, the model reduction method based on the single natural modal resonance theory [11] is employed. The used method is originated from the classical model reduction techniques $[12,13]$ but has great advantage in reducing the total sizes of system model. The reduced order model owing to the used method of this paper has a smaller of DOFs, usually one or two DOFs in many applications.

The outline of this paper is as follows. The reduction method based on the single natural mode resonance theory is briefly introduced in first section. Some applicable conditions for the presented method are also involved in this section. An illustratable example of mass-spring-damper system with ten DOFs is examined, and some important results are obtained and discussed in the subsequent section. The results obtained from the presented method will be verified with numerically solving the original system. Conclusions are drawn in the last section.

\section{Theoretical Fundamentals and Formulations}

The motion equations of an $n$-DOFs dynamical system with local cubic nonlinearities can be expressed in matrix form as:

$$
[M]\{\ddot{x}\}+[C]\{\dot{x}\}+[K]\{x\}+\left\{F_{N}(x)\right\}=\left\{F_{d}(t)\right\},
$$


where $\{x\}$ is the $n$-vector of physical coordinates, $[M],[C]$, and $[K]$ are $n \times n$ mass, damping, and stiffness matrices, respectively, $\left\{F_{N}(x)\right\}$ is the $n$-vector of cubic nonlinear applied force, and $\left\{F_{d}(t)\right\}$ is the vector of time-dependent external excitations.

In this paper, the mass matrix $[M]$ and the stiffness matrix $[C]$ are assumed to be symmetric, the external excitations $\left\{F_{d}(t)\right\}$ are harmonic, and the damping matrix in (2.1) is proportional to the mass and/or stiffness matrices for system, that is,

$$
[C]=a[M]+b[K],
$$

where the parameters $a$ and $b$ are constants.

The homogeneous undamped equation

$$
[M]\{\ddot{x}\}+[K]\{x\}=0
$$

leads to eigenvalue or spectral matrix $[\Lambda]$ and eigenvector matrix $[\Phi]$.

The spectral matrix $[\Lambda]$ is given by

$$
[\Lambda]=\operatorname{diag}\left(\left[\begin{array}{llll}
\omega_{1} & \omega_{2} & \cdots & \omega_{n}
\end{array}\right]\right),
$$

and the eigenvector matrix $[\Phi]$ is expressed as

$$
[\Phi]=\left[\begin{array}{llll}
\phi_{1} & \phi_{2} & \cdots & \phi_{n}
\end{array}\right]
$$

The eigenpairs $\left(\omega_{i}, \phi_{i}\right)$ denote the $i$ th modal frequency and modal shape, $i=1,2, \ldots, n$.

Introduce the transformation

$$
\{x\}=[\Phi]\{q\},
$$

where $\{q\}$ is the $n$-vector of normal mode coordinates.

Substituting (2.6) into (2.1) yields

$$
[M][\Phi]\{\ddot{q}\}+[C][\Phi]\{\dot{q}\}+[K][\Phi]\{q\}+\left\{F_{N}([\Phi]\{q\})\right\}=\left\{F_{d}(t)\right\} \text {. }
$$

Multiplying (2.7) by the transpose $\left[\Phi^{T}\right]$, one obtains

$$
\left[\Phi^{T}\right][M][\Phi]\{\ddot{q}\}+\left[\Phi^{T}\right][C][\Phi]\{\dot{q}\}+\left[\Phi^{T}\right][K][\Phi]\{q\}+\left[\Phi^{T}\right]\left\{F_{N}([\Phi]\{q\})\right\}=\left[\Phi^{T}\right]\left\{F_{d}(t)\right\} .
$$

The eigenvector matrix satisfies the following orthogonality properties with respect to the mass and stiffness matrix

$$
\left[\Phi^{T}\right][M][\Phi]=[I], \quad\left[\Phi^{T}\right][K][\Phi]=[\Lambda]
$$

where $[I]$ is unit matrix. 
In case of Rayleigh damping, the damping term $\left[\Phi^{T}\right][C][\Phi]$ in (2.8) can be written to

$$
[\bar{C}]=\left[\Phi^{T}\right][C][\Phi]=\operatorname{diag}\left(\left[\begin{array}{llll}
2 \xi_{1} \omega_{1} & 2 \xi_{2} \omega_{2} & \cdots & 2 \xi_{n} \omega_{n}
\end{array}\right]\right)
$$

where $\xi_{i}$ is the $i$ th modal damping coefficient.

Using (2.9) and (2.10), then (2.8) is transformed to

$$
\{\ddot{q}\}+[\bar{C}]\{\dot{q}\}+[\Lambda]\{q\}+\left[\Phi^{T}\right]\left\{F_{N}(q)\right\}=\left[\Phi^{T}\right]\left\{F_{d}(t)\right\}
$$

or

$$
\ddot{q}_{i}+2 \xi_{i} \omega_{i} \dot{q}_{i}+\omega_{i}^{2} q_{i}+\sum_{j=1}^{n} \phi_{j i} F_{N j}\left(\begin{array}{llll}
q_{1} & q_{2} & \cdots & q_{n}
\end{array}\right)=\sum_{j=1}^{n} \phi_{j i} F_{d j}(t)
$$

Comparing (2.1) with (2.11) or (2.12), one can find that they are equivalent in mathematics. Unlike (2.1), the terms in (2.11) or (2.12) expect the nonlinear force are uncouple.

Equation (2.11) or (2.12) gives the modal response equations of system. For a MDOFs system, the total response of system is a sum of the response of all natural modes. Generally, higher modes contribute less toward the total response of system. Thus, an approximate response can be determined by some lower modes, that is, (2.6) can be approximately written in

$$
\{x\} \approx \sum_{i=1}^{l} \phi_{i} q_{i}
$$

where the variable $l$ denotes the number of lower modes and is always no more than the number of degrees of freedom of the original system.

Obviously, (2.12) and (2.13) describe a reduced order model in the form of mode coordinates with $l$-DOFs for the original model. Based on the above model, one can quickly get some important results in quantity. It is the key of classical model reduction technique.

After observation and investigation on engineering problems for a long time, Prof. Zheng discloses that actual engineering systems are always controlled by a few modes, almost one or two modes in many situations. He further points out that only a single mode (corresponding to the resonance mode) is maybe leading as system enters into resonance state. His finding has a general significance. For many engineering problems, one cares more for dynamics of system under resonance state. Undoubtedly, this finding powers and strengthens traditional model reduction techniques. In this paper, we follow his studies and apply his theory to investigate nonlinear dynamics of a nonlinear MDOFs system under super/sub harmonic resonance conditions.

Suppose that only the $j$ th mode of system is leading and the other modes are minor as the super or sub harmonic resonance of the $j$ th mode takes place. Thus, the total response 
of system is approximately determined by neglecting the contribution of the nonresonance modes and only remaining the component owed to the resonance mode, that is, to let

$$
\{x\} \approx\left\{\phi_{j}\right\} q_{j} .
$$

Substituting (2.14) into (2.11) or (2.12), one can get the equations of reduced model in the form of resonance modal coordinate

$$
\ddot{q}_{j}+2 \xi_{j} \omega_{j} \dot{q}_{j}+\omega_{j}^{2} q_{j}+\sum_{k=1}^{n} \phi_{k j} F_{N k}\left(q_{j}\right)=\sum_{k=1}^{n} \phi_{k j} F_{d k}(t)
$$

and the equations in the form of non-resonance modal coordinates

$$
\ddot{q}_{i}+2 \xi_{i} \omega_{i} \dot{q}_{i}+\omega_{i}^{2} q_{i}+\sum_{k=1}^{n} \phi_{k i} F_{N k}\left(q_{j}\right)=\sum_{k=1}^{n} \phi_{k i} F_{d k}(t)
$$

Considering cubic nonlinearities and harmonic excitation (e.g., cosine excitation) in system, (2.15) can be compactly rewritten to

$$
\ddot{q}_{j}+2 \varepsilon \mu \dot{q}_{j}+\omega_{j}^{2} q_{j}+\varepsilon \alpha q_{j}^{3}=F_{0} \cos (\omega t)
$$

where $\varepsilon$ is small parameter $\mu$, and $\alpha, F_{0}, \omega$, are constants. $\omega$ denotes the frequency of excitation.

Obviously, (2.17) is a single DOF equation. One can easily get its solution by the analytic methods or numerical methods. Then, one can obtaine the approximate response of the system, associated with (2.14).

As described in the above text, the theory presented by Zheng [11] is powerful. However, this theory is semiempirical and is not strictly formulated in mathematics. After investigations, the author of this paper finds that the theory may be generally available under the following conditions.

(i) The nature frequencies of system have sparsely in distribution. It means that only the resonance mode is leading while the non-resonance modes contribute less toward the total response of system at resonance.

(ii) The resonance mode should not interact with other non-resonance modes. It implies that internal resonance does not occur.

(iii) For multifrequency excitation, combination resonance may occur, but the response of combination resonance is not preponderant compared with that of interested resonance mode.

Now, we use the above theory to seek an approximate solution. In weak vibration, an approximate steady solution of first order for (2.17) is supposed to be in the form

$$
q_{j}(t, \varepsilon)=q_{j 0}\left(T_{0}, T_{1}\right)+\varepsilon q_{j 1}\left(T_{0}, T_{1}\right)+\cdots .
$$


Substituting (2.18) into (2.17) and equating the coefficients of the same power of small parameter $\varepsilon$, one obtains

$$
\begin{gathered}
D_{0}^{2} q_{j 0}+\omega_{j}^{2} q_{j 0}=F_{0} \cos \left(\omega T_{0}\right), \\
D_{0}^{2} q_{j 1}+\omega_{j}^{2} q_{j 1}=-2 D_{0} D_{1} q_{j 0}-2 \mu D_{0} q_{j 0}-\alpha q_{j 0}^{3} .
\end{gathered}
$$

The general solution of (2.19) can be expressed in the form

$$
q_{j 0}=A\left(T_{1}\right) e^{i \omega_{j} T_{0}}+\Lambda e^{i \omega T_{0}}+c c,
$$

where $\Lambda=F_{0} /\left(2\left(\omega_{j}^{2}-\omega^{2}\right)\right)$, cc stands for the complex conjugate of the preceding terms.

Substituting (2.21) into (2.20) yields

$$
\begin{aligned}
D_{0}^{2} q_{j 1}+\omega_{j}^{2} q_{j 1}= & -\left[2 i \omega_{j}\left(D_{1} A+\mu A\right)+6 \alpha A \Lambda^{2}+3 \alpha A^{2} \bar{A}\right] e^{i \omega_{j} T_{0}} \\
& -\alpha\left[A^{3} e^{3 i \omega_{j} T_{0}}+\Lambda^{3} e^{3 i \omega T_{0}}+3 A^{2} \Lambda e^{i\left(2 \omega_{j}+\omega\right) T_{0}}+3 \bar{A}^{2} \Lambda e^{i\left(\omega-2 \omega_{j}\right) T_{0}}\right] \\
& -\alpha\left[3 A \Lambda^{2} e^{3 i\left(\omega_{j}+2 \omega\right) T_{0}}+3 A \Lambda^{2} e^{i\left(\omega_{j}-2 \omega\right) T_{0}}\right]-\Lambda\left(-2 i \mu \omega+3 \alpha \Lambda^{2}+6 \alpha A \bar{A}\right) e^{i \omega T_{0}} .
\end{aligned}
$$

Two cases of resonance are considered next: superharmonic and subharmonic.

\subsection{Superharmonic Resonance}

In this case, we put

$$
3 \omega=\omega_{j}+\varepsilon \sigma
$$

where $\sigma$ is turning parameter.

Inserting (2.23) into (2.22), the condition for the elimination of secular terms in (2.22) is

$$
2 i \omega_{j}\left(D_{1} A+\mu A\right)+6 \alpha A \Lambda^{2}+3 \alpha A^{2} \bar{A}+\alpha \Lambda^{3} e^{i \sigma T_{1}}=0
$$

To this order, $A$ is considered to be a function of $T_{1}$ only. Then, substituting the polar form

$$
A=\left(\frac{1}{2}\right) a\left(T_{1}\right) e^{i \theta T_{1}}
$$


into (2.24) and equating the real and imaginary parts, one gets

$$
\begin{gathered}
\dot{a}=-\mu a-\frac{\alpha \Lambda^{3}}{\omega_{j}} \sin \left(\sigma T_{1}-\theta\right), \\
a \dot{\theta}=\frac{\alpha}{\omega_{j}}\left[3 a\left(\Lambda^{2}+\frac{a^{2}}{8}\right)+\Lambda^{3} \cos \left(\sigma T_{1}-\theta\right)\right] .
\end{gathered}
$$

Introduce $\gamma=\sigma T_{1}-\theta$, then (2.26) can be written to

$$
\begin{gathered}
\dot{a}=-\mu a-\frac{\alpha \Lambda^{3}}{\omega_{j}} \sin \gamma \\
a \dot{\theta}=\frac{\alpha}{\omega_{j}}\left[3 a\left(\Lambda^{2}+\frac{a^{2}}{8}\right)+\Lambda^{3} \cos \gamma\right]
\end{gathered}
$$

Let $\dot{a}=\dot{\theta}=0$, the stable period solutions $a_{s}$ and $\gamma_{s}$ are satisfied with

$$
\begin{gathered}
\mu a_{s}=-\frac{\alpha \Lambda^{3}}{\omega_{j}} \sin \gamma_{s} \\
{\left[\sigma-\frac{3 \alpha}{\omega_{j}}\left(\Lambda^{2}+\frac{a_{s}^{2}}{8}\right)\right] a_{s}=\frac{\alpha \Lambda^{3}}{\omega_{j}} \cos \gamma_{s}}
\end{gathered}
$$

Thus, the steady solution is

$$
q_{j}(t)=\Lambda \cos (\omega t)+\varepsilon a_{s} \cos \left(3 \omega t+\gamma_{s}\right)
$$

Considering (2.28), the frequency response curve for superharmonic resonance is

$$
\left[\mu^{2}+\left(\sigma-\frac{3 \alpha \Lambda^{2}}{\omega_{j}}-\frac{3 \alpha a_{s}^{2}}{8 \omega_{j}}\right)^{2}\right] a_{s}^{2}=\frac{\alpha^{2} \Lambda^{6}}{\omega_{j}^{2}} .
$$

With (2.29) and (2.14), one can get the stable superharmonic response of system (2.1). Similarly, combinated using (2.30) and (2.14), the frequency response characteristic of original system is obtained.

\subsection{Sub Harmonic Resonance}

In this case, we take

$$
\omega=3 \omega_{j}+\varepsilon \sigma .
$$




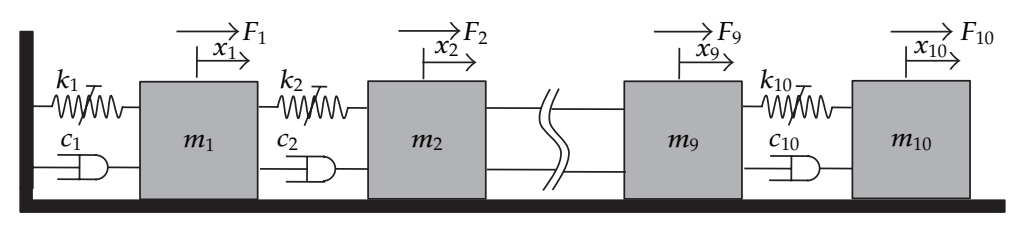

Figure 1: Schematic of a mass-damping-spring system.

Inserting (2.31) into (2.22), the condition for the elimination of secular terms is

$$
2 i \omega_{j}\left(D_{1} A+\mu A\right)+6 \alpha A \Lambda^{2}+3 \alpha A^{2} \bar{A}+3 \alpha \bar{A}^{2} \Lambda e^{i \sigma T_{1}}=0
$$

Reused the rule in the case of superharmonic resonance, the steady state solution is

$$
q_{j}(t)=\Lambda \cos (\omega t)+\varepsilon a_{s} \cos \left(\frac{\omega t}{3}+\gamma_{s}\right)
$$

where $a_{s}$ and $\gamma_{s}$ are governed by

$$
\begin{gathered}
\mu=-\frac{3 \alpha \Lambda}{4 \omega_{j}} a_{s} \sin \gamma_{s}, \\
\sigma-\frac{9 \alpha}{\omega_{j}}\left(\Lambda^{2}+\frac{a_{s}^{2}}{8}\right)=\frac{9 \alpha \Lambda}{4 \omega_{j}} a_{s} \cos \gamma_{s} .
\end{gathered}
$$

The frequency response curve is

$$
9 \mu^{2}+\left(\sigma-\frac{9 \alpha \Lambda^{2}}{\omega_{j}}-\frac{9 \alpha}{8 \omega_{j}} a_{s}^{2}\right)^{2}=\frac{81 \alpha^{2} \Lambda^{2}}{16 \omega_{j}^{2}} a_{s}^{2} .
$$

With (2.33), (2.35), and (2.14), one can get steady state solution of system (2.1) and the nonlinear frequency response characteristic.

\section{Numerical Examples}

In this section, the mechanical model for discrete mass-damping-spring system is shown in Figure 1, which consists of ten mass blocks $m_{i}$, supported by nonlinear springs and linear dampers with coefficient $c_{i}$. The excitation $F_{i}$ on the $i$ th mass block is assumed to be cosine, with amplitude $f_{i}$, frequency $\Omega$, and initial phase $\theta_{i}, i=1,2, \ldots, n$. The physical coordinate $x_{i}$ donates absolute displacement of the $i$ th mass block, which is measured from its equilibrium position.

The restoring force of the $i$ th spring is determined by

$$
F_{i}\left(u_{i}\right)=k_{i} u_{i}+\alpha_{i} u_{i}^{3}
$$

where $k_{i}$ and $\alpha_{i}$ are linear and nonlinear coefficients, $u_{i}$ denotes the deformation of spring. 
Utilizing the Newton second law, the equations governing the motion of the system are then, given in matrix form as

$$
[M]\{\ddot{X}\}+[C]\{\dot{X}\}+[K]\{X\}=\{F(t)\}+\left\{F_{N}(X)\right\},
$$

where the mass matrix $[M]$ is

$$
[M]=\operatorname{diag}\left(\left[\begin{array}{llll}
m_{1} & m_{2} & \cdots & m_{10}
\end{array}\right]\right),
$$

the stiffness matrix $[K]$ is

$$
[K]=\left[\begin{array}{ccccc}
k_{1}+k_{2} & -k_{2} & & & \\
-k_{2} & k_{2}+k_{3} & -k_{3} & & \\
& \ddots & \ddots & \ddots & \\
& & -k_{9} & k_{9}+k_{10} & -k_{10} \\
& & & -k_{10} & k_{10}
\end{array}\right]
$$

the excitation force $\{F(t)\}$ is

$$
\{F(t)\}=\left[\begin{array}{c}
f_{1} \cos \left(\Omega t+\theta_{1}\right) \\
f_{2} \cos \left(\Omega t+\theta_{2}\right) \\
\vdots \\
f_{10} \cos \left(\Omega t+\theta_{10}\right)
\end{array}\right]
$$

the nonlinear component of the restoring force $\left\{F_{N}(X)\right\}$ is

$$
\left\{F_{N}(X)\right\}=\left[\begin{array}{c}
\alpha_{2}\left(x_{2}-x_{1}\right)^{3}-\alpha_{1} x_{1}^{3} \\
\alpha_{3}\left(x_{3}-x_{2}\right)^{3}-\alpha_{2}\left(x_{2}-x_{1}\right)^{3} \\
\vdots \\
\alpha_{10}\left(x_{10}-x_{9}\right)^{3}-\alpha_{9}\left(x_{9}-x_{8}\right)^{3} \\
-\alpha_{10}\left(x_{10}-x_{9}\right)^{3}
\end{array}\right] .
$$

In case of Rayleigh damping, the damping matrix $[C]$ is

$$
[C]=a[M]+b[K]
$$

The parameters used in this simulation are

$$
\begin{array}{r}
k=100000, \quad m_{i}=1000 \mathrm{~kg}, \quad \alpha_{i}=25 k^{2}, \quad i=1,2, \ldots, n, \quad f_{i}=0, \quad i \neq 10, \\
f_{10}=50 \mathrm{~N}, \quad a=0.018, \\
\quad b=0.002, \quad k_{1}=k_{2}=5 \mathrm{kN} / \mathrm{m}, \quad k_{3}=k_{4}=4 k \mathrm{~N} / \mathrm{m}, \quad k_{5}=k_{6}=3 k \mathrm{~N} / \mathrm{m}, \\
k_{7}=k_{8}=2 k \mathrm{~N} / \mathrm{m}, \quad k_{9}=k_{10}=k \mathrm{~N} / \mathrm{m} .
\end{array}
$$


Table 1: The first four natural frequencies.

\begin{tabular}{lc}
\hline Mode & Freq. (rad/s) \\
\hline 1 & 2.7502 \\
2 & 6.6523 \\
3 & 10.8492 \\
4 & 15.0825 \\
\hline
\end{tabular}

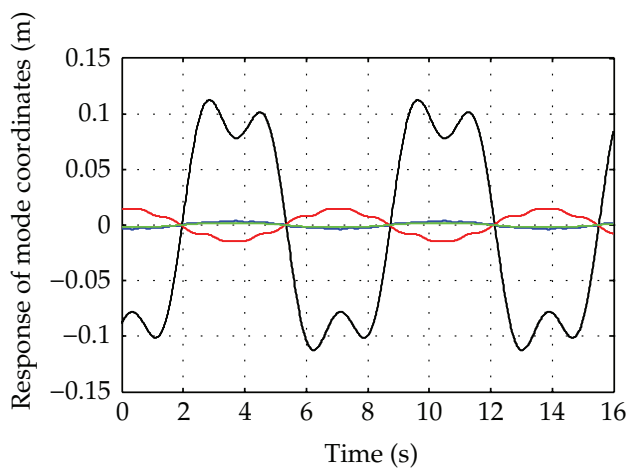

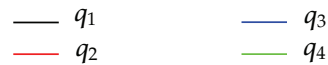

(a)

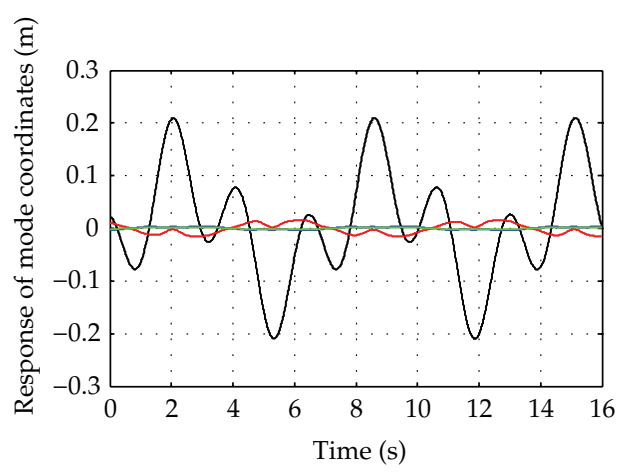

$\begin{array}{ll}q_{1} & -q_{3} \\ q_{2} & -q_{4}\end{array}$

(c)
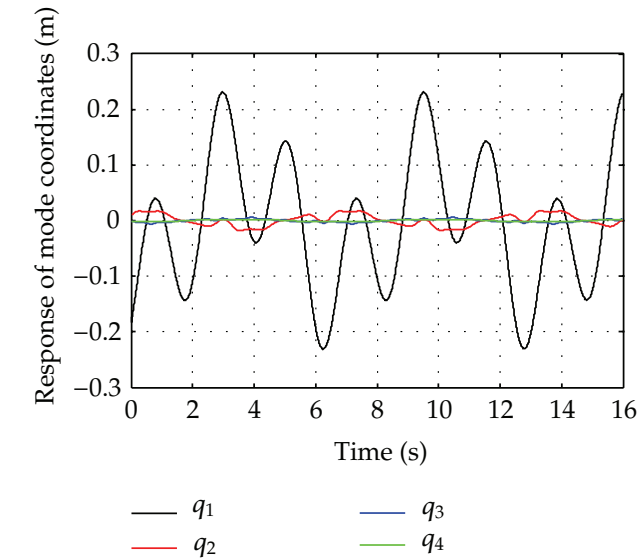

(b)
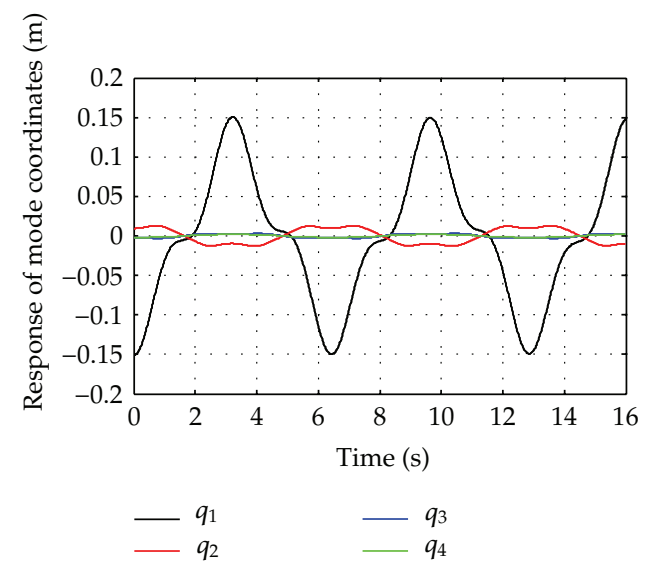

(d)

Figure 2: The time response of the first four modes for different excitation frequency $\Omega$ : black line, first mode; red line, second mode; blue line, third mode; green line, fourth mode. (a) $\Omega=0.9267$, (b) and (c) $\Omega=0.9617$, and (d) $\Omega=0.9767(\mathrm{rad} / \mathrm{s})$.

\subsection{The Vibration Characteristic Analysis}

The eigenfrequency analysis for the dynamic system discovers the fundamental vibration characteristic of the system, for example, resonance frequency and vibration shape. For this purpose, we first perform the eigenfrequency analysis. This problem is equivalent to solve eigenvalues of undamper, free vibration equations of (3.2). The QR method is used to find its eigenvalues. The first four natural frequencies are reported in Table 1. 


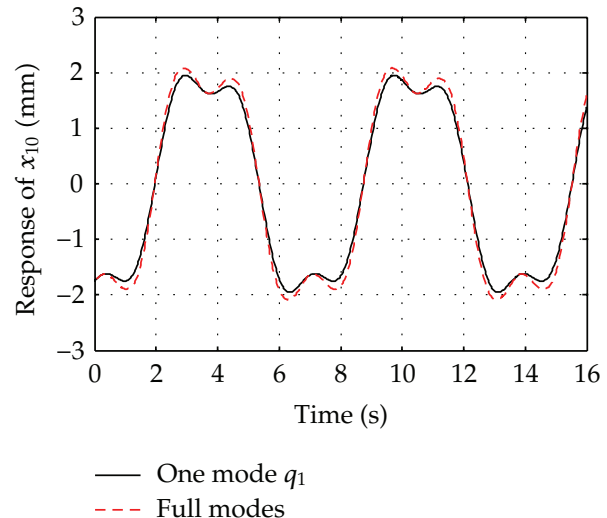

(a)

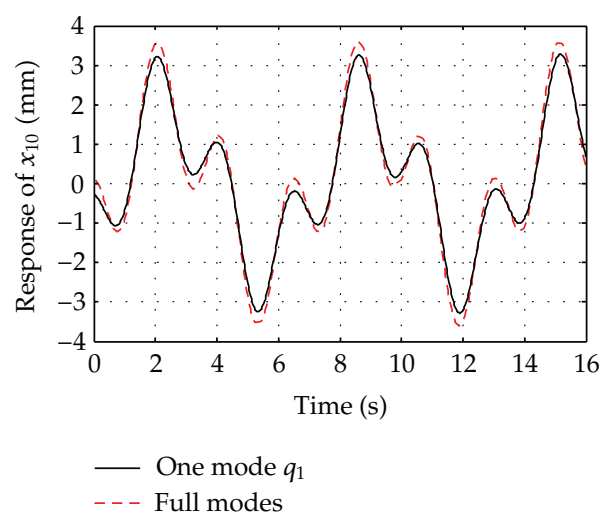

(c)

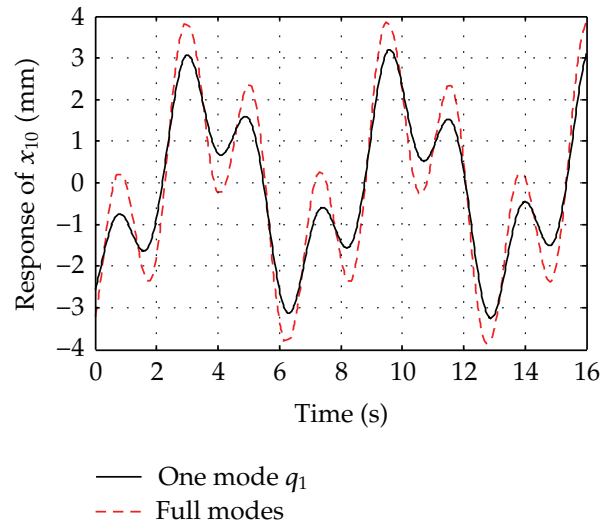

(b)

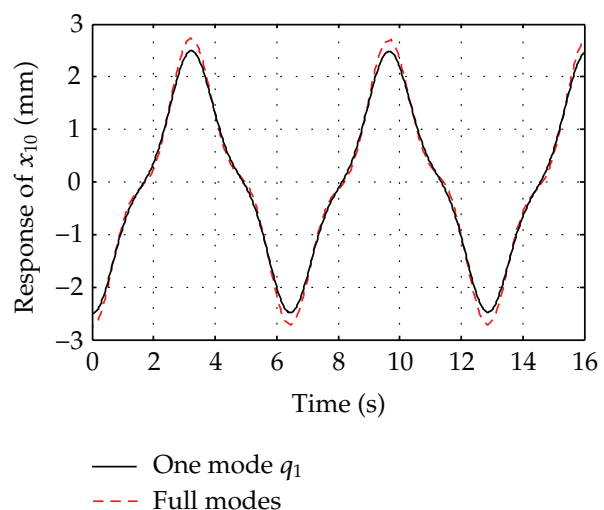

(d)

Figure 3: Comparison between the response obtained from the reduced model for resonance mode $q_{1}$ and numerical integration of original model using full modes: - , single mode; ---, full modes, (a) $\Omega=0.9267$, (b) and (c) $\Omega=0.9617$, (d) $\Omega=0.9767$ ( rad/s).

In terms of nonlinear vibration theory, the system subjected to harmonic excitation with its frequency $\Omega$ approaching one third of or three times of any of natural frequency of the system may enters into resonance state: the superharmonic resonance or sub harmonic resonance. The dynamic response of the system under superharmonic or sub harmonic resonance condition is studied in the next section.

\subsection{Super Harmonic Resonance}

In this section, we use the presented method to investigate the super harmonic resonance. Only the superharmonic resonance corresponding to the first two natural frequencies is considered.

\subsubsection{The Case for $3 \Omega \approx \omega$}

In the case of excitation frequency near one third of the first natural frequency, we first carry out an analysis of the vibration response of all modes with a series of given frequencies. 


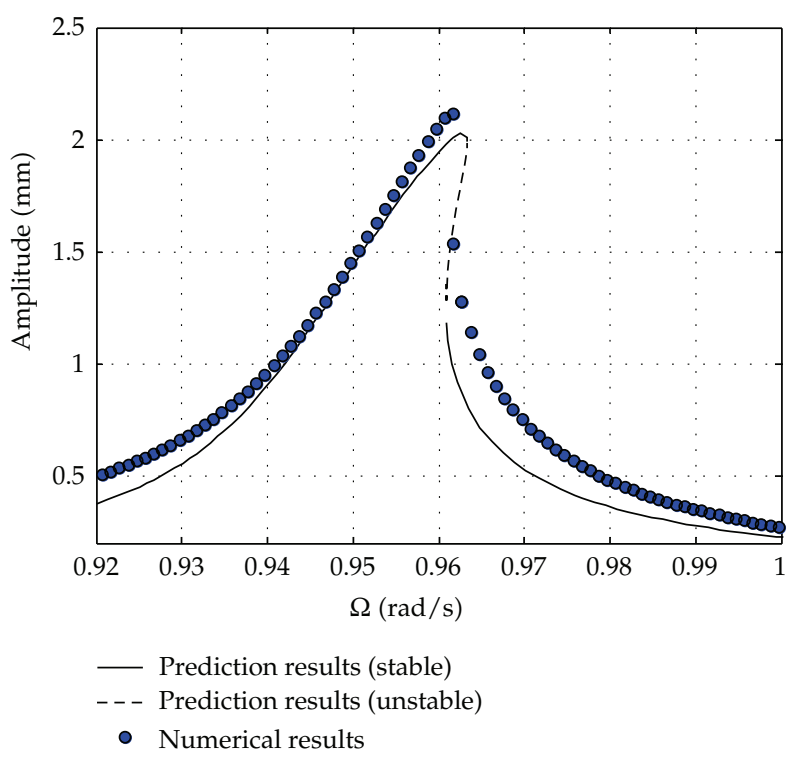

Figure 4: The frequency response curve for $3 \Omega \approx \omega_{1}$.

To perform it, (3.2) is transformed to the one in the form of mode coordinates. The modes' responses at different excitation frequencies are then obtained from the numerical integration for the modal equation by using the fifth fourth order Runge-Kutta-Fehlberg (RKF) method with adaptive step size. In simulation, the excitation frequency is taken to be $0.9267 \mathrm{rad} / \mathrm{s}, 0.9617 \mathrm{rad} / \mathrm{s}$, and $0.9767 \mathrm{rad} / \mathrm{s}$, respectively. Numerical results are plotted in Figure 2. Figure 2 illustrates the time response curve of the first four modes of system. Figures 2(a), 2(b), and 2(d) are for the excitation frequency $0.9267 \mathrm{rad} / \mathrm{s}, 0.9617 \mathrm{rad} / \mathrm{s}$, and $0.9767 \mathrm{rad} / \mathrm{s}$, respectively. Figures $2(\mathrm{~b})$ and 2 (c) are for the same excitation frequency but different integral initial conditions. Clearly shown in Figure 2, the response of the first mode is leading, while the response of other modes is weak as the excitation frequency is around one-third of the first natural frequency.

From the data curve plotted in Figure 2, one can infer the total response of system that is governed by the first mode. Based on the presented method, the motion equation for the first mode $q_{1}$ can be approximately expressed as

$$
\ddot{q}_{1}+2 \varepsilon_{1} \mu_{1} \dot{q}_{1}+\omega_{1}^{2} q_{1}+\varepsilon_{1} \beta_{1} q_{1}^{3}=f_{1} \cos (\Omega t),
$$

where the parameters are $\varepsilon_{1}=0.01, \mu_{1}=1.656, \beta_{1}=2230$, and $f_{1}=0.829$.

The numerical integration of (3.9) by RKF method yields the response of the first mode. Considering the law given by (2.14), one may obtain the approximate response of the original system (3.2). The approximate response is plotted by solid line in Figure 3. To validate the approximate response, we compared it with the result obtained from directly numerical integration of (3.2), seen in Figure 3. Figure 3 shows the steady response of displacement of the tenth mass block of the system regard with time. The dashed line is for the integration result of (3.2), and the solid line is for the approximate result generated by the presented method. Figures 3(a), 3(b), and 3(d) are for the excitation frequency $\Omega$ equaling to $0.9267 \mathrm{rad} / \mathrm{s}, 0.9617 \mathrm{rad} / \mathrm{s}$, and $0.9767 \mathrm{rad} / \mathrm{s}$, respectively. The data in Figure 3 demonstrate 


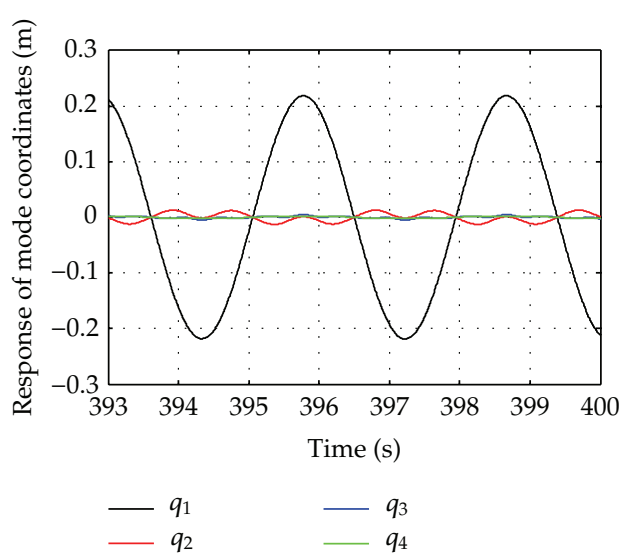

(a)

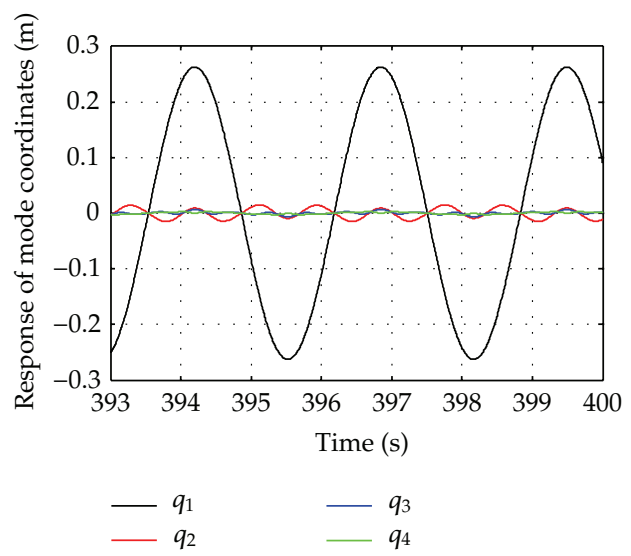

(c)

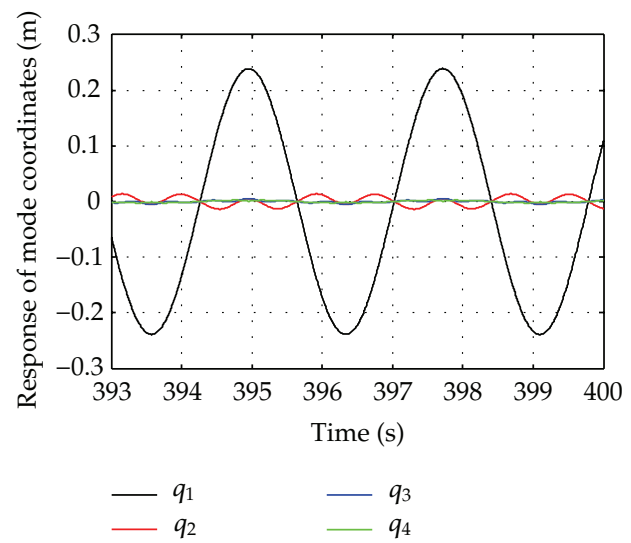

(b)

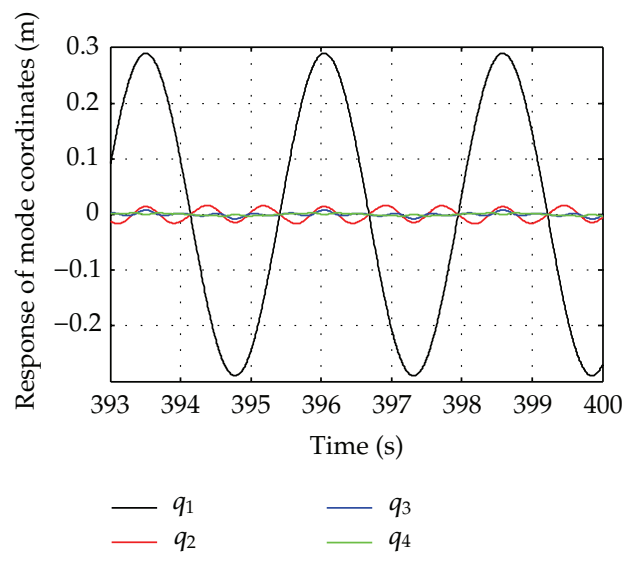

(d)

Figure 5: Comparison of the time response of the first four modes for different excitation frequency in $\mathrm{rad} / \mathrm{s}$ (a) $\Omega=2.175$, (b) $\Omega=2.275$, (c) $\Omega=2.375$, and (d) $\Omega=2.475$.

that the dynamic response is surely mainly controlled by the first mode of the system at the first superharmonic resonance state.

During the simulation, the system is shown to be sensitive to initial conditions, seen in Figures 3(b) and 3(c). Figures 3(b) and 3(c) are for the same excitation frequency 0.9617 $\mathrm{rad} / \mathrm{s}$ but different initial conditions. Obviously, Figures 3(b) and 3(c) are corresponding to two different stable steady solution. The data curve in Figures 3(b) and 3(c) imply that there are two steady period solutions in the region of frequency around $0.9617 \mathrm{rad} / \mathrm{s}$.

In order to determine the frequency region for coexistence of multiple period solutions for the system, the approximate frequency response characteristic equation is derived from (3.9), seen in (2.30). The frequency response characteristic curve is plotted by the solid line in Figure 4 . Figure 4 shows that the displacement response amplitude of the tenth mass block of system varies along with the excitation frequency $\Omega$. As shown in Figure 4 , there exist two steady period solutions in the region from $0.9608 \mathrm{rad} / \mathrm{s}$ to $0.9634 \mathrm{rad} / \mathrm{s}$, and the jump in the frequency response curve appeared at frequency $0.9608 \mathrm{rad} / \mathrm{s}$ and $0.9634 \mathrm{rad} / \mathrm{s}$. These results 


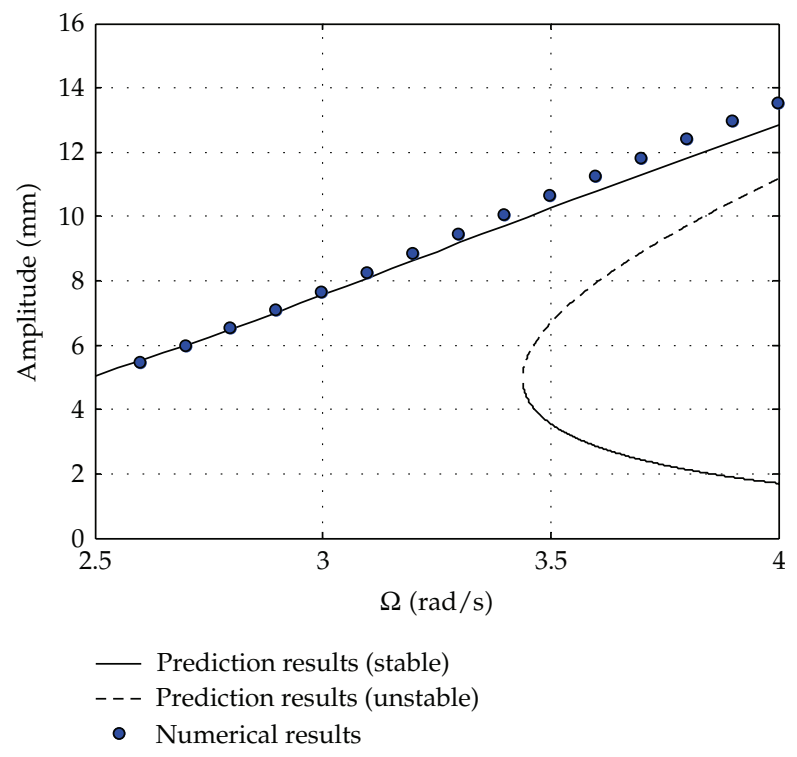

Figure 6: The frequency response curve for $3 \Omega \approx \omega_{2}$.

give a qualitative prediction for the nonlinear dynamic behaviors of the system (3.2) under superharmonic resonance of the first mode.

We calculate the integration of (3.2) numerically with frequency sweeping at a step of 0.001 in either upward or downward direction. In terms of nonlinear oscillation theory, the response not only includes the fundamental frequency $\Omega$, but also frequency components at $\Omega, 3 \Omega, 5 \Omega$, and so on. In weak vibration, only two frequency components are mainly observed to be included in the response of the system, that is, the frequencies $\Omega$ and $3 \Omega$. In the response, the component of frequency $\Omega$ is forced, and the component of frequency $3 \Omega$ originated from nonlinear resonance. We examine the response and separated the component of frequency equaling to third time for the excitation frequency from the total response. The results are plotted in Figure 4 by dotted points. Clearly in Figure 4, the prediction results match well with the numerical results to large extent.

\subsubsection{The case for $3 \Omega \approx \omega_{2}$}

Similarly, we first study the mode response of the system around the superharmonic resonance of the second mode. The comparison between the responses of the first four modes is shown in Figure 5. Figures 5(a)-5(d) are for the excitation frequency $2.175 \mathrm{rad} / \mathrm{s}$, $2.275 \mathrm{rad} / \mathrm{s}, 2.375 \mathrm{rad} / \mathrm{s}$, and $2.475 \mathrm{rad} / \mathrm{s}$, respectively. Results shown in Figure 5 indicate that the response of the first mode is much stronger than that of other modes besides the nominal resonance mode-the second mode. The phenomenon is caused by the primary resonance of the first mode. In this case, the excitation frequency is near the first natural frequency. The nonlinearities make an easy way for the occurrence of the first primary resonance other than the second superharmonic resonance. Thus, the superharmonic resonance of the second mode is quenched by the first mode's primary resonance. The dynamic response of system is actually determined by the first mode in this case. Using the presented method, one can get 


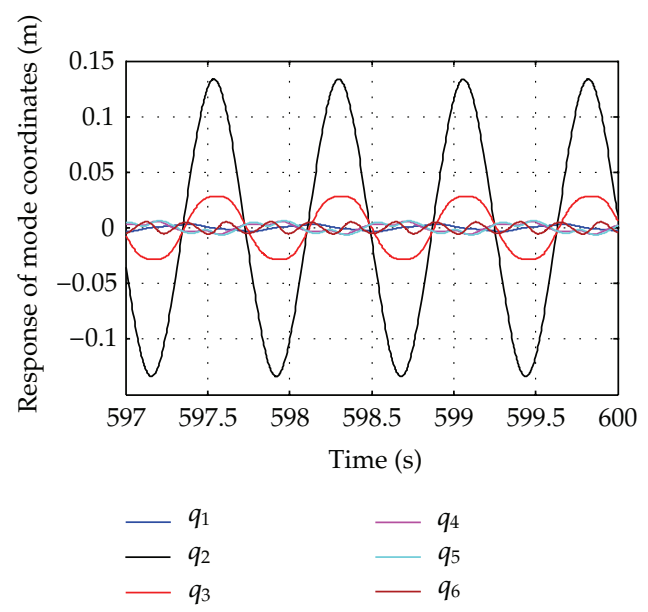

(a)

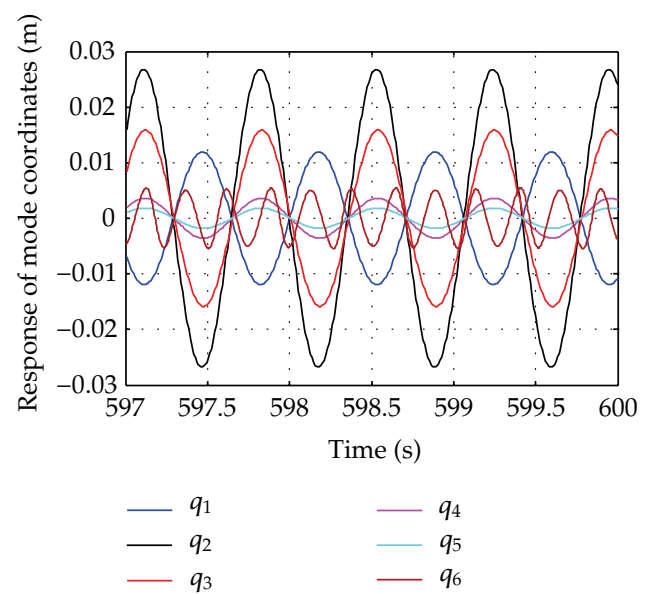

(c)
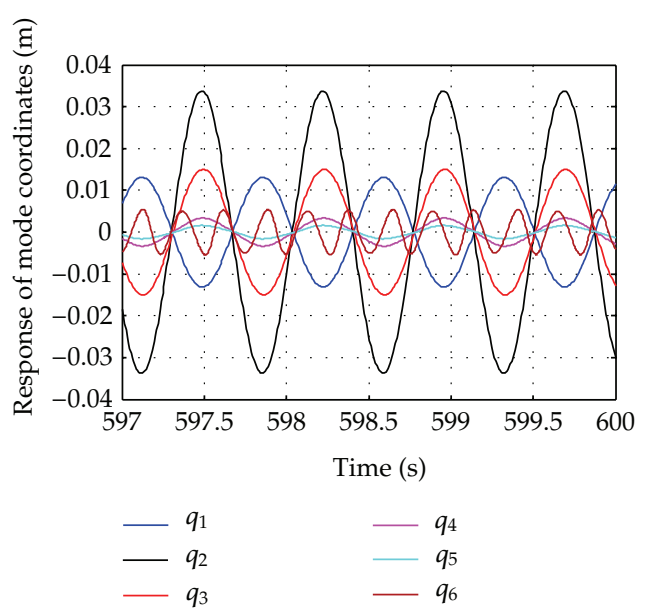

(b)

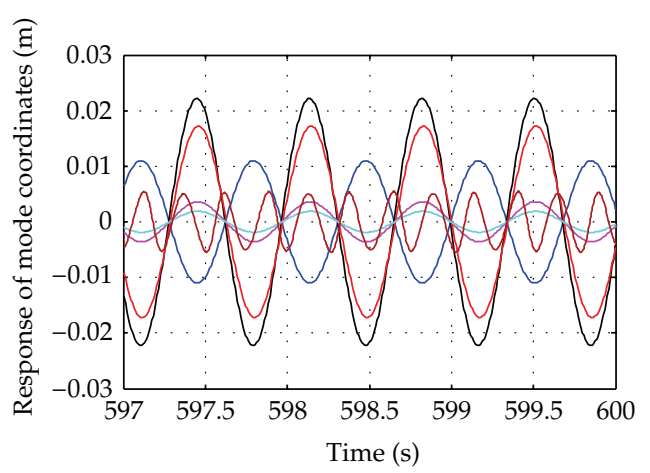

(d)

Figure 7: Comparison of the time response of the first six mode at different excitation frequency $\Omega(\mathrm{rad} / \mathrm{s})$ : (a) $\Omega=8.16$, (b) $\Omega=8.26$ (c) $\Omega=8.86$, (d) $\Omega=9.16$.

the motion equation governed by the first mode at the first primary resonance. Considering the primary resonance is not the topic of this paper, the dynamic equation for the first mode is not definitely given, and it will be further investigated in other studies. Figure 6 is the frequency response curve for $3 \Omega \approx \omega_{2}$. The circles in Figure 6 denote the response amplitude of the tenth mass block of system obtained from the numerical integration of (3.2). The solid line and the dashed line in Figure 6 are plotted by the dynamic model for the first mode.

\subsection{Sub Harmonic Resonance}

In this section, the dynamic response of the system under sub harmonic resonance conditions is examined. Two cases are just involved, $\Omega \approx 3 \omega_{1}$ and $\Omega \approx 3 \omega_{2}$. 

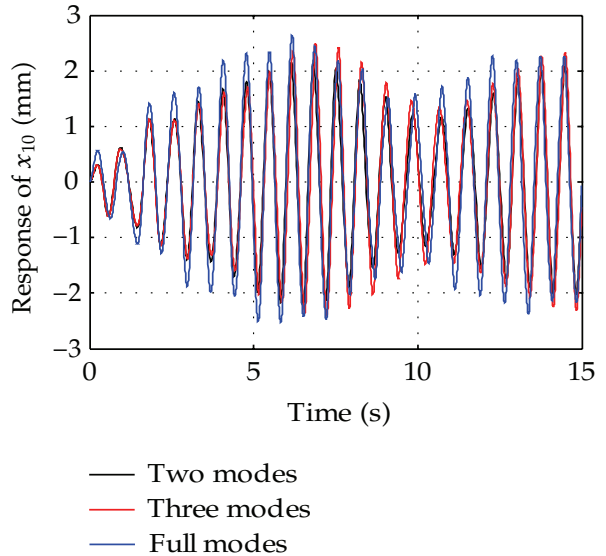

(a)

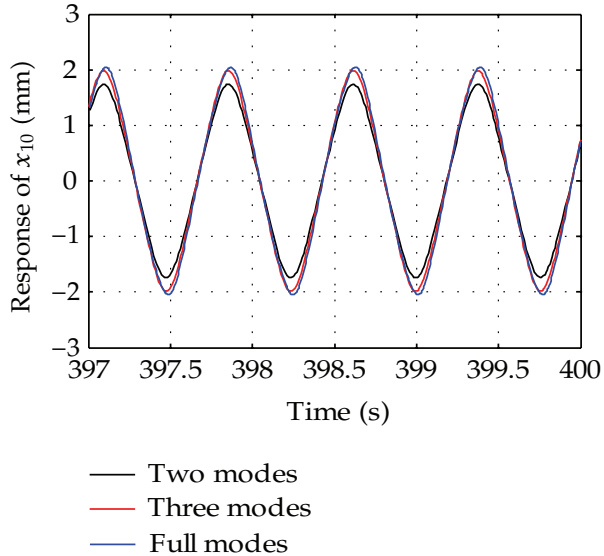

(b)

Figure 8: Comparison of the response of $x_{10}$ obtained from the reduced model using different modes at excitation frequency $\Omega=8.26 \mathrm{rad} / \mathrm{s}$ : (a) transient response; (b) steady response.

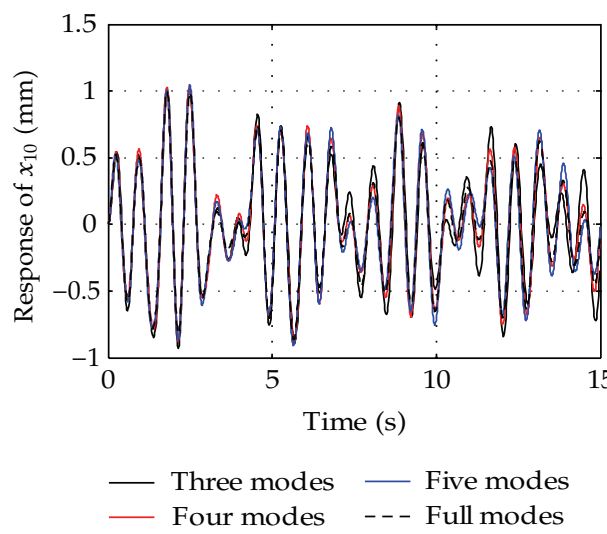

(a)

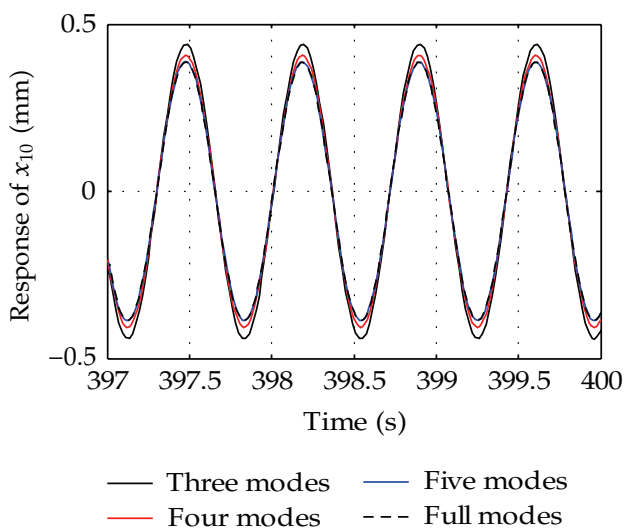

(b)

Figure 9: Comparison of the response of $x_{10}$ obtained from the reduced model using different modes at excitation frequency $\Omega=8.86 \mathrm{rad} / \mathrm{s}$ : (a) transient response; (b) steady response.

\subsubsection{The Case for $\Omega \approx 3 \omega_{1}$}

The system with multiple degrees of freedom always has multiple natural modes. As the system forced by the excitation, multiple modes are sometimes excited, seen in Figure 7. Figure 7 shows the first six modes' steady response around the excitation frequency near the three time of the first natural frequency. The values of excitation frequency for Figures $7(\mathrm{a})-7(\mathrm{~d})$ are $8.26,8.56,8.86$, and 9.16, respectively. As shown in Figure 7, the response of the second mode is the strongest. The contribution of the third mode to the total response of the system cannot be ignored. The first mode nominally acts as the resonance mode, but its response is not leading instead. In this case, one can approximate the response of system by multiple modes which contribute larger toward the response of system. For instance, we approximate the response of the system using the two modes: second mode 


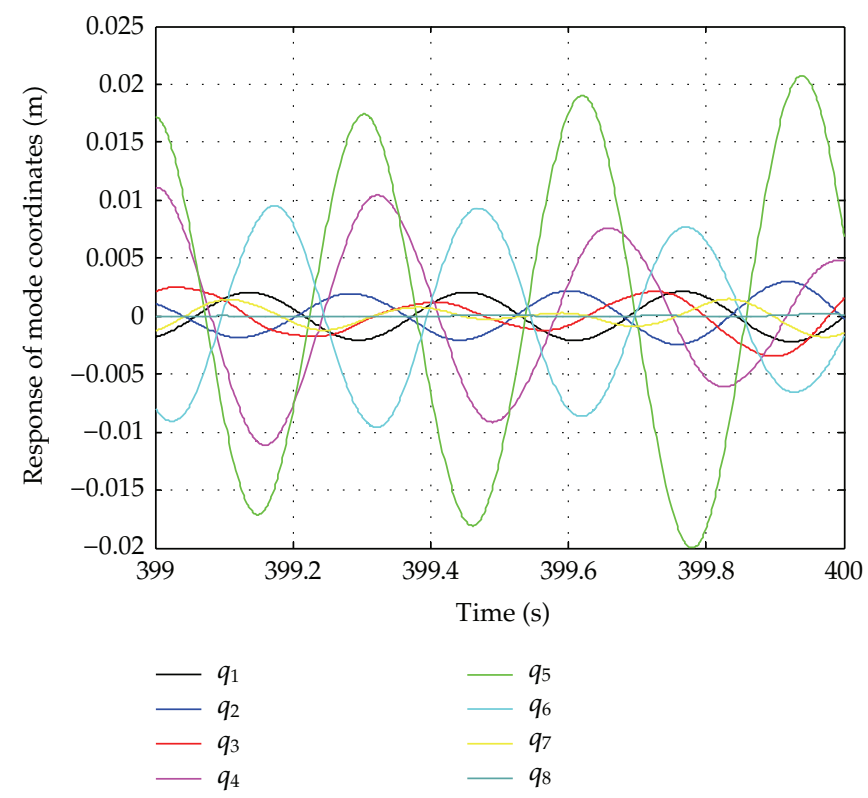

Figure 10: Comparison of the response of the first eight modes at $\Omega=20 \mathrm{rad} / \mathrm{s}$.

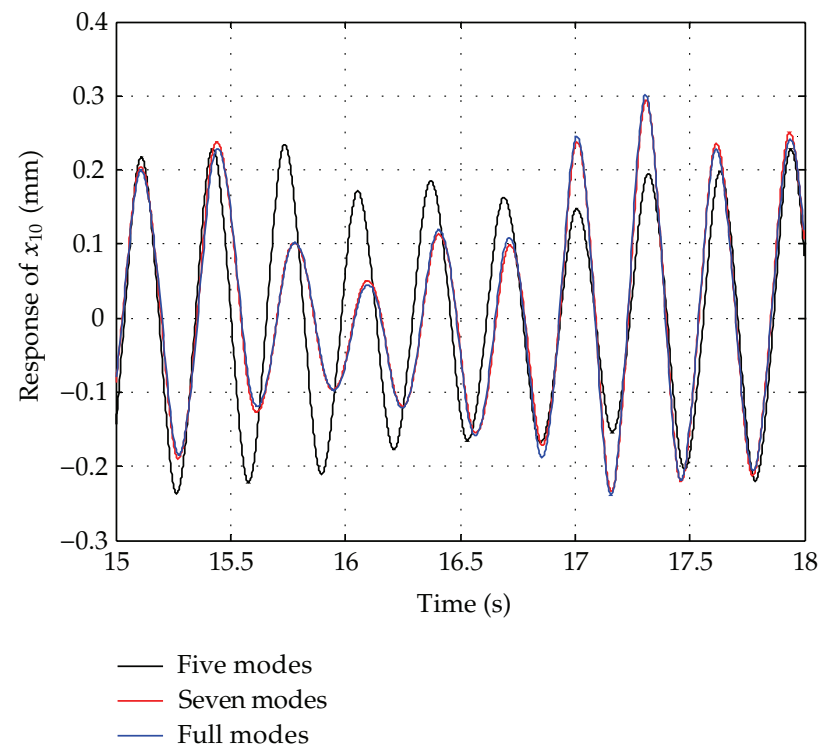

Figure 11: Comparison of the response of coordinate $x_{10}$ from the different reduced models $\Omega=20 \mathrm{rad} / \mathrm{s}$.

and third mode. The numerical results are plotted by black line in Figures 8(a) and 8(b). Figures $8(\mathrm{a})$ and $8(\mathrm{~b})$ are for the transient and steady response of the tenth mass block in system for given excitation frequency $\Omega=8.26 \mathrm{rad} / \mathrm{s}$, respectively. In this simulation, we also examine the response of system by the combination of the first three modes and full modes. The corresponding results are plotted in Figures $8(\mathrm{a})$ and $8(\mathrm{~b})$ by red line and blue 


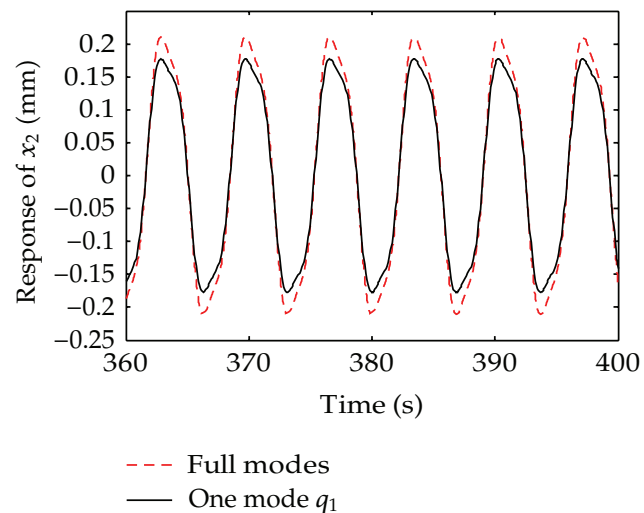

(a)

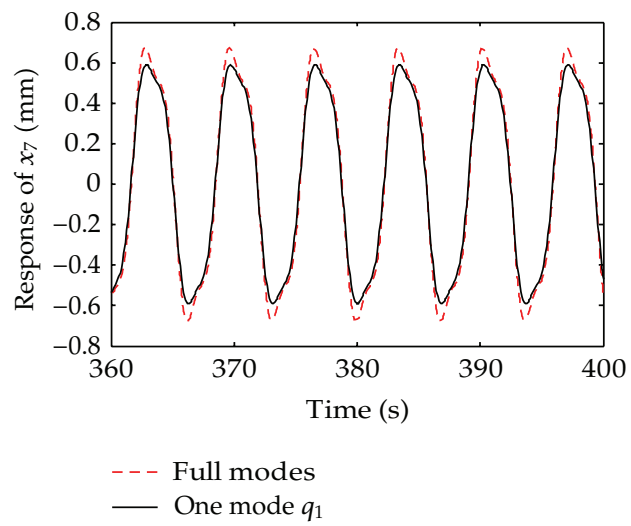

(c)

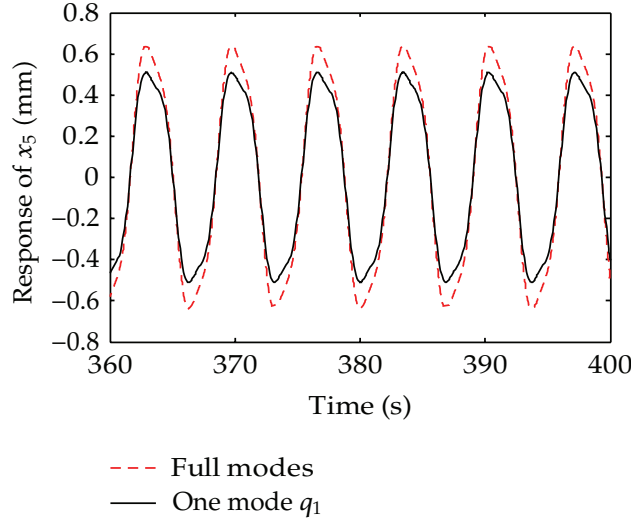

(b)

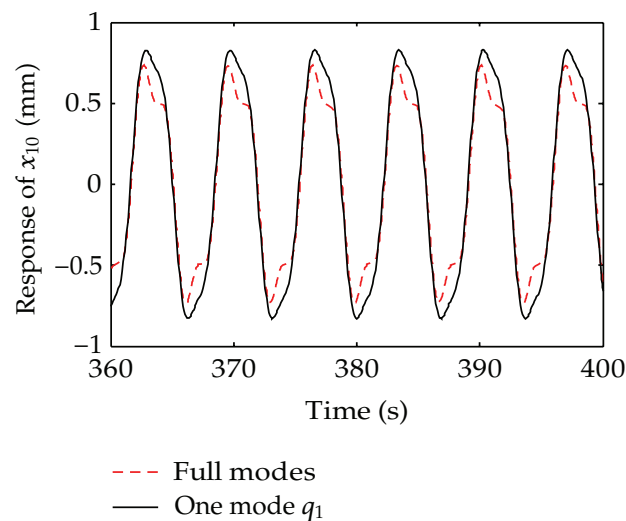

(d)

Figure 12: Time response of different physical coordinates for case A.

line, respectively. From Figures 8(a) and 8(b), we can conclude that the results determined by the first three modes approximate well the results from the numerical integration of (3.2).

Similarly, we approximate the response of system by the different combination of modes at frequency $\Omega=8.86 \mathrm{rad} / \mathrm{s}$. Results show the response is complex, and the approximation is obtained by more modes. Take as the response of the tenth mass block for example. Its transient and steady response are plotted in Figures 9(a) and 9(b). From the data curve in Figures 9(a) and 9(b), the results determined by five modes are in good agreements with the results obtained from the full modes. In this simulation, the used modes are the first five modes.

\subsubsection{The Case for $\Omega \approx 3 \omega_{2}$}

Now, we investigate the dynamic behavior of the system around the excitation frequency equaling to three times of the second natural frequency. Results show that the dynamic behavior is very complex in high frequency vibration. Figure 10 shows the first eight modes' response for excitation frequency $\Omega=20 \mathrm{rad} / \mathrm{s}$. Clearly, multiple modes are excited the same time and contribute to the total response of system. We use the first five modes, the 


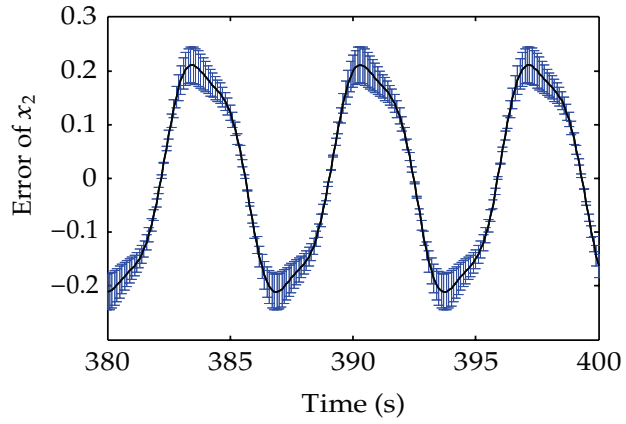

(a)

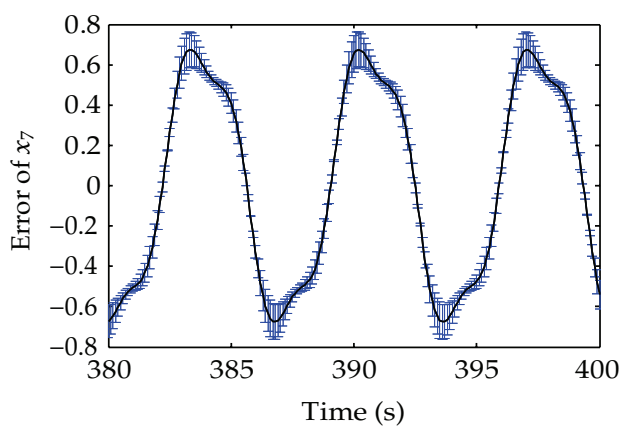

(c)

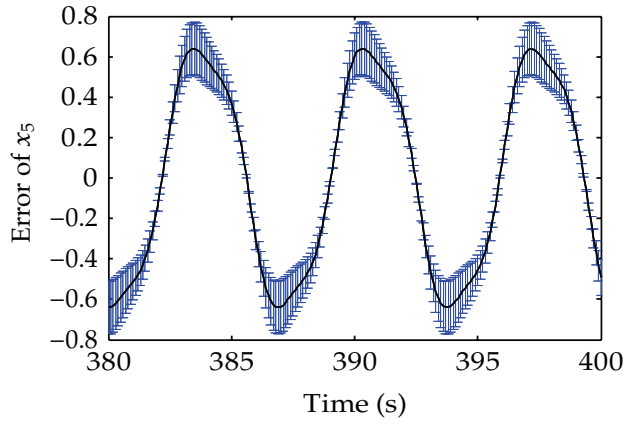

(b)

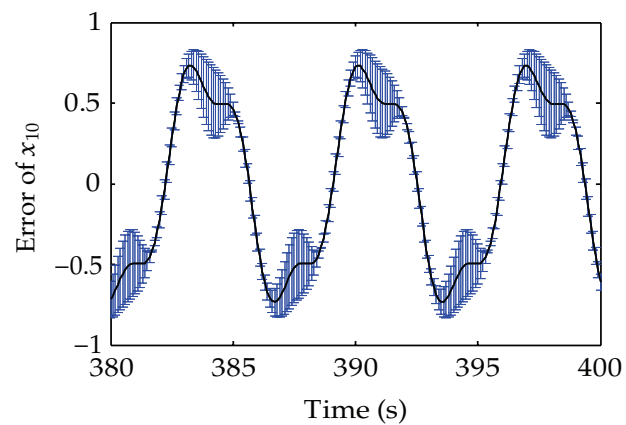

(d)

Figure 13: Analysis of error bars for case A.

first seven modes, and full modes to examine the dynamic response of system, respectively. Results are plotted in Figure 11. Obviously in Figure 11, the results determined by the first seven modes approximate well the results obtained from the original model. To approximate the dynamical response of high frequency, more modes should be used.

\subsection{Error Analysis}

To quantitatively evaluate the approximate results, we carry out an error analysis. For a general purpose, we consider the following three cases:
(A) $f_{5}=50, f_{i}=0, i \neq 5, \Omega=\omega_{1} / 3=0.917$;
(B) $f_{3}=20, f_{9}=50, f_{i}=0, i \neq 3,9, \Omega=\omega_{1} / 3=0.917$;
(C) $f_{8}=50, f_{i}=0, i \neq 8, \Omega=3 \omega_{1}=8.2505$.

\subsubsection{Case $A$}

Figure 12 shows the time response of different physical coordinates. The corresponding error analysis between the approximate solutions and the referred solutions obtained from direct integration of (3.2) is given in Figure 13. As shown in Figures 12 and 13, the approximate solutions capture main dynamical behaviors of system (3.2), although local differences occur as the vibration responses of system arrive at the peak or low amplitude. 


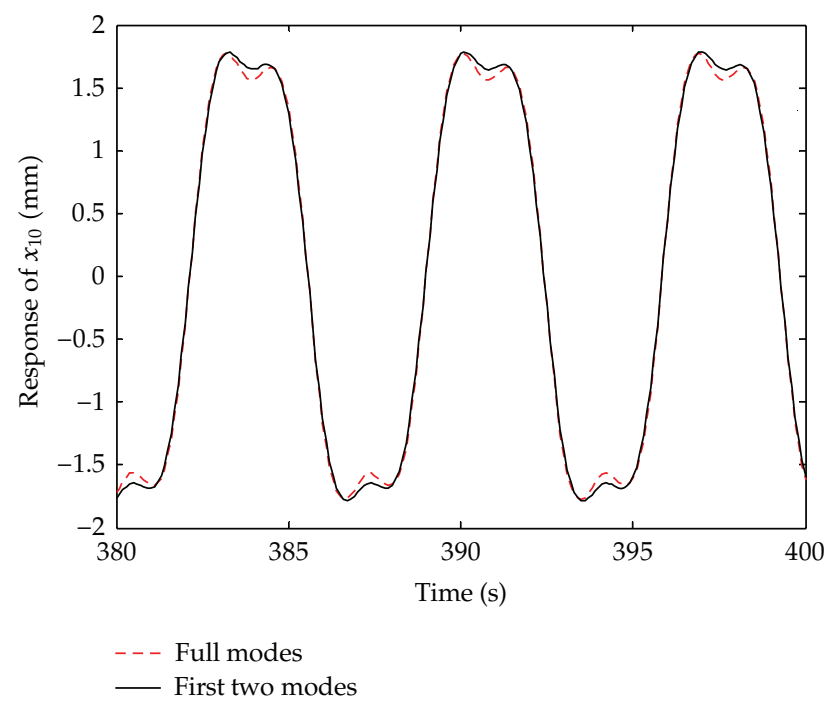

Figure 14: Time response of $x_{10}$ for case B.

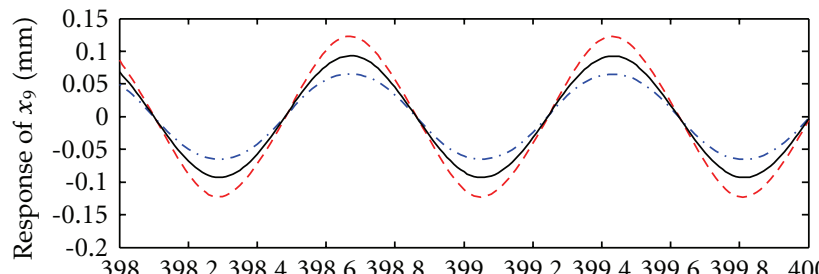

- - - Full modes

. -. - First three modes

— First four modes

(a)

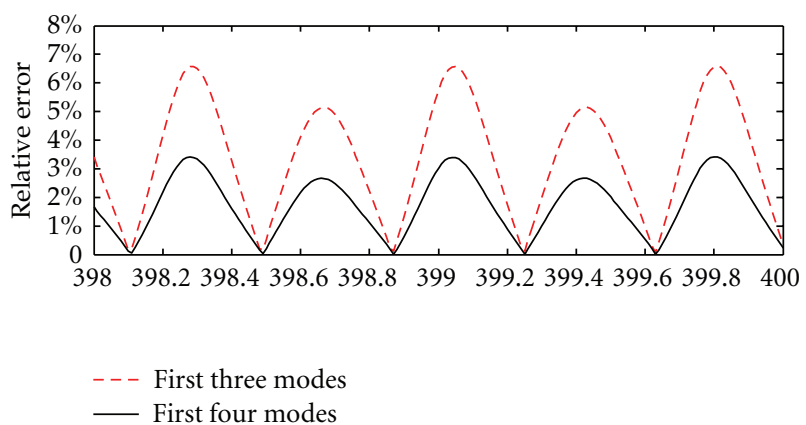

(b)

Figure 15: Error analysis for case C. 
Table 2: Relative errors analysis for case B.

\begin{tabular}{lcccccccccc}
\hline Time (s) & 380 & 380.4 & 381.5 & 382.1 & 382.5 & 383 & 383.9 & 384.4 & 385 & 385.4 \\
\hline Rel. error & $4.3 \%$ & $5.4 \%$ & $2.7 \%$ & $1.0 \%$ & $3.8 \%$ & $1.1 \%$ & $5.1 \%$ & $1.7 \%$ & $3.3 \%$ & $5.6 \%$ \\
\hline
\end{tabular}

\subsubsection{Case B}

From Figures 12 and 13, we can conclude that high modes affect the local dynamical properties of system. To correct it, we use multiple modes to approximate the system solutions. Results are presented in Figure 14 and Table 2. In this case, the first two modes are considered. Figure 14 depicts the time response of physical coordinate $x_{10}$. In Figure 14, red dashed line represents the solutions from the calculation of integration for (3.2), black solid line denotes the approximate solutions with two modes. The relative errors between the two results are accounted for in Table 2.

\subsubsection{Case C}

In this case, the response of physical coordinate $x_{9}$ is examined. We employ the first three modes and the first four modes to approximate its time response curve, respectively. Results are plotted in Figure 15. The analysis of error further illuminates the vibration of high frequency is very complex. To obtain the approximate solutions with enough accuracy, more modes should be considered.

\section{Conclusions}

The response of a MDOFs' system with local cubic nonlinearities under super- and subharmonic resonance conditions is investigated in this paper. The responses of modes of linearized system are first examined by numerical integration. By comparisons of the responses of all modes, the leading modes are found, which control the whole response of system. In the presence of a single leading mode, the single natural modal resonance theory is used to generate a reduced dynamical model with only a single dof. The qualitative and quantitative results are obtained. By comparing them with the numerical results from the integration of the dynamic equations of system, the approximation results by the presented method are, to large extent, in agreements with the numerical results. The nonlinear frequency response characteristic is included in this paper. In weak vibration, the analytical expression of frequency response equation is derived from the reduced model. The jump and the region for coexistence of multiple steady state solutions are successfully predicted. In the case of coaction of multiple modes, the approximate response of system is obtained by the reduced models described by multiple different modes. Results show that the response of higher frequency is very complex. To get good approximation, more modes are combinated used. This paper provides us with a new way to get fast an approximate super-/subharmonic solution of a MDOFs system with local nonlinearities at resonance state in both quality and quantity.

\section{Acknowledgment}

The author would like to thank Professor Zheng ZhaoChang and Professor Ren GeXue for their help in this work. 


\section{References}

[1] M. K. Yazdi, H. Ahmadian, A. Mirzabeigy, and A. Yildirim, "Dynamic analysis of vibrating systems with nonlinearities," Communications in Theoretical Physics, vol. 57, pp. 183-187, 2012.

[2] I. Kovacic and M. J. Brennan, The Duffing Equation: Nonlinear Oscillators and Their Behaviour, John Wiley \& Sons, 2011.

[3] A. I. Manevich and L. I. Manevitch, The Mechanics of Nonlinear Systems with Internal Resonances, Imperial College Press, 2005.

[4] J. C. Ji and C. H. Hansen, "On the approximate solution of a piecewise nonlinear oscillator under super-harmonic resonance," Journal of Sound and Vibration, vol. 283, no. 1-2, pp. 467-474, 2005.

[5] A. M. Elnaggar and A. F. El-Basyouny, "Harmonic, subharmonic, superharmonic, simultaneous sub/super harmonic and combination resonances of self-excited two coupled second order systems to multi-frequency excitation," Acta Mechanica Sinica, vol. 9, no. 1, pp. 61-71, 1993.

[6] M. Eissa and A. F. El-Bassiouny, "Analytical and numerical solutions of a non-linear ship rolling motion," Applied Mathematics and Computation, vol. 134, no. 2-3, pp. 243-270, 2003.

[7] T. C. Kim, T. E. Rook, and R. Singh, "Super- and sub-harmonic response calculations for a torsional system with clearance nonlinearity using the harmonic balance method," Journal of Sound and Vibration, vol. 281, no. 3-5, pp. 965-993, 2005.

[8] C. Duan and R. Singh, "Super-harmonics in a torsional system with dry friction path subject to harmonic excitation under a mean torque," Journal of Sound and Vibration, vol. 285, no. 4-5, pp. 803834, 2005.

[9] R. W. Hamming, Numerical Methods for Scientists and Engineers, Dover Publications, 1987.

[10] B. W. Bader, Tensor-Krylov Methods for Solving Large-scale Systems of Nonlinear Equations, University of Colorado, 2003.

[11] Z. C. Zheng, "On the intrinsic simple connection of linear with nonlinear system-the quantity and qualitative analysis of large scale multiple DOF nonlinear systems," in Proceedings of the National Structure Dynamics Conference, pp. 1-13, Nanchang, China, 2008.

[12] M. I. Friswell, J. E. T. Penny, and S. D. Garvey, "Using linear model reduction to investigate the dynamics of structures with local non-linearities," Mechanical Systems and Signal Processing, vol. 9, no. 3, pp. 317-328, 1995.

[13] R. H. B. Fey, D. H. van Campen, and A. de Kraker, "Long term structural dynamics of mechanical systems with local nonlinearities," Journal of Vibration and Acoustics, vol. 118, no. 2, pp. 147-153, 1996. 


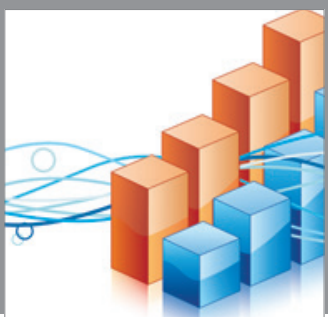

Advances in

Operations Research

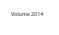

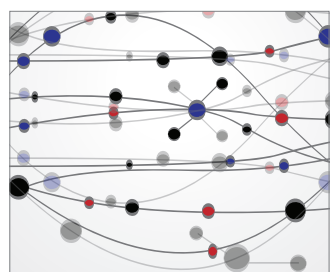

\section{The Scientific} World Journal
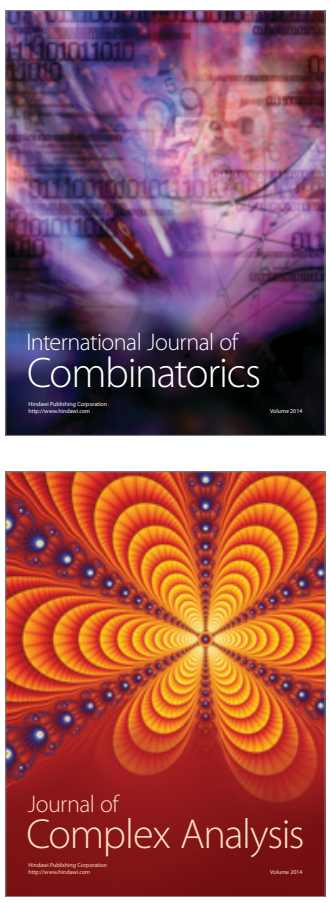

International Journal of

Mathematics and

Mathematical

Sciences
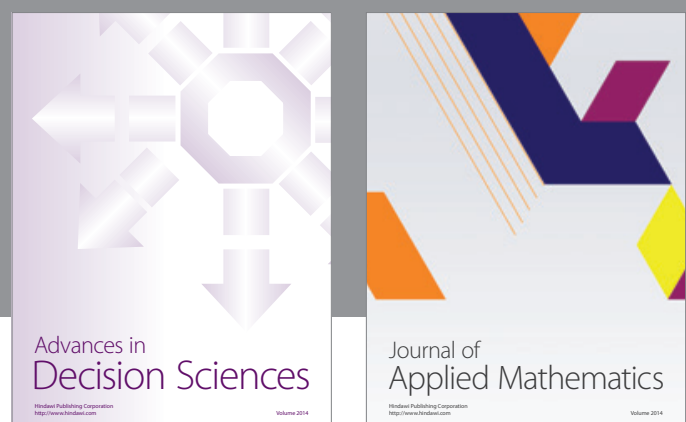

Journal of

Applied Mathematics
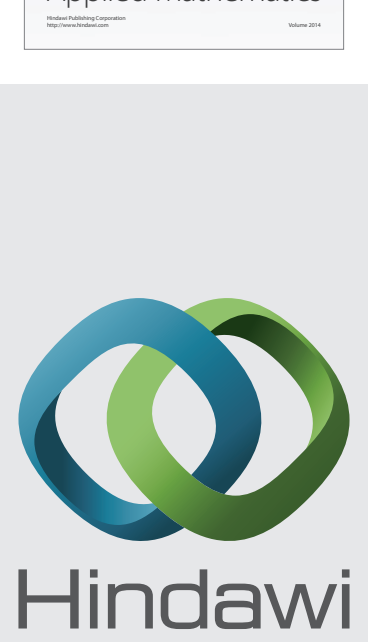

Submit your manuscripts at http://www.hindawi.com
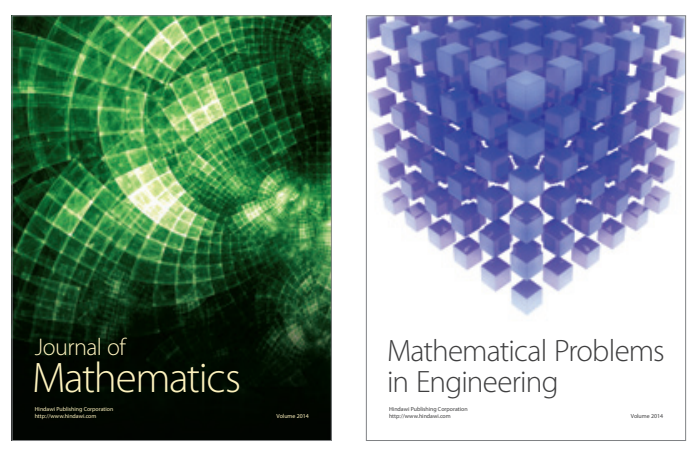

Mathematical Problems in Engineering
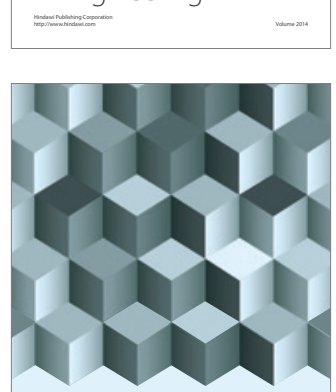

Journal of

Function Spaces
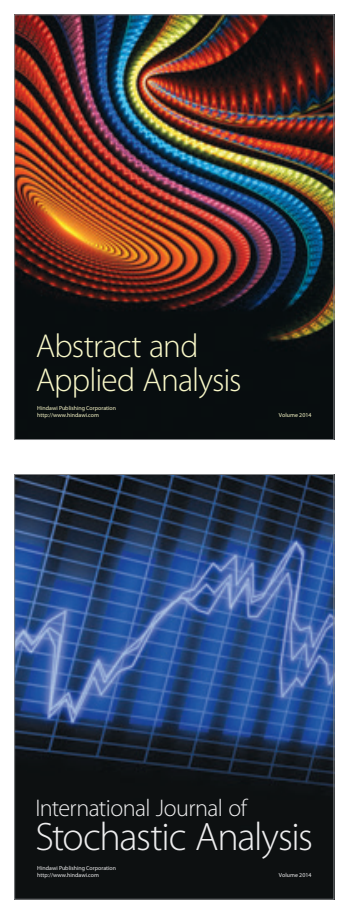

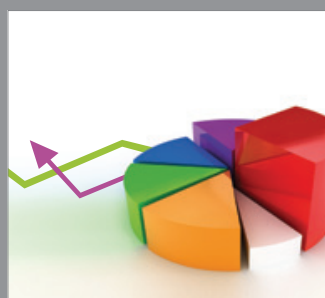

ournal of

Probability and Statistics

Promensencen
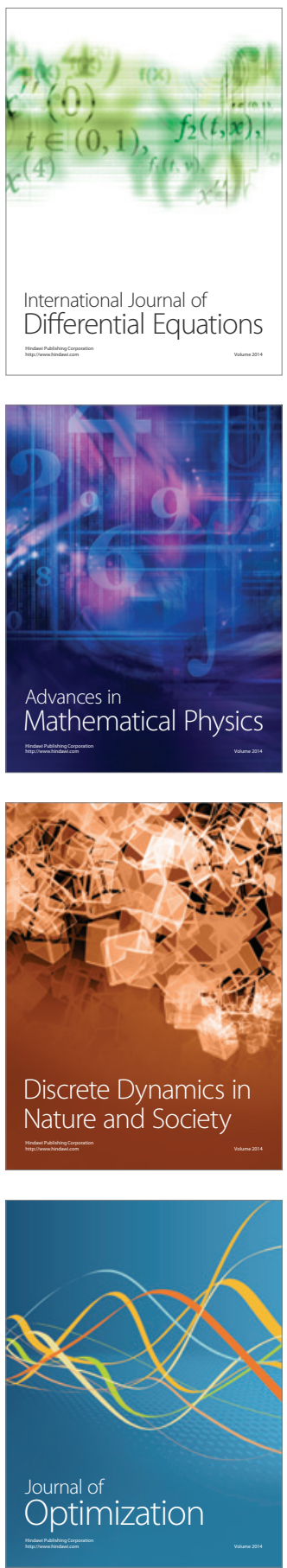